\title{
Research on MAC Protocols in Cluster-Based Ad Hoc Networks
}

\author{
Yutao Liu $\mathbb{D}^{1},{ }^{1}$ Yue Li, ${ }^{2}$ Yimeng Zhao, ${ }^{1}$ and Chunhui Zhang ${ }^{1}$ \\ ${ }^{1}$ The 54th Research Institute of CETC, Shijiazhuang 050081, China \\ ${ }^{2}$ Naval Research Institute, Beijing 100161, China \\ Correspondence should be addressed to Yutao Liu; liuyutao_7@163.com
}

Received 29 January 2021; Revised 24 February 2021; Accepted 10 March 2021; Published 30 March 2021

Academic Editor: Xin Liu

Copyright (c) 2021 Yutao Liu et al. This is an open access article distributed under the Creative Commons Attribution License, which permits unrestricted use, distribution, and reproduction in any medium, provided the original work is properly cited.

Mobile ad hoc networks can be widely used in many scenes, for example, military communication, emergency communication, and $5 \mathrm{G}$ wide area coverage as well as ultradense network scenes. A cluster-based network can decrease network overhead effectively and then constitute a large-scale network through extending network scale by clustering and cascading. In view of multiservice simultaneous transmission demand for small-scale dense networking scene and large-scale extended networking scene, a MAC protocol based on scheduling of cluster head is proposed, which can avoid collision and retransmission between nodes effectively, and then the network synchronizing in intercluster and intracluster as well as the time-slot scheduling is solved fast and efficiently. A network synchronization algorithm based on clock spread of the sponsorship node is proposed to achieve network self-synchronizing, and a modified schedule-tree algorithm based on cluster head and exactly sponsorship node is proposed to decrease network overhead and to increase network fairness, then MAC protocol based on scheduling of cluster head is verified by simulated analysis. Finally, a dynamic gateway selection algorithm based on link stability is proposed in this paper to decrease intercluster communication control overhead and improve intercluster communication reliability.

\section{Introduction}

Mobile ad hoc networks, usually, are referred to MANET $[1,2]$. According to the discussion of [3-5], MANET is a wireless mobile network that consists of a group of mobile nodes with wireless transceiver communication equipment. MANET is not dependent on base infrastructure and is always a temporary network; the mobile nodes in the network can be communicated from their wireless transceiver equipment. When out of communication range, the nodes in the MANET can realize multihop relaying communication with the help of middle nodes. With the advantages of not depending on fixed infrastructure, self-organization, and multihop, MANET becomes more and more popular in military communication and emergency communication. In addition, ad hoc network is one of the key technologies for $5 \mathrm{G}$ wide area coverage and ultradense network scenes. With the help of ad hoc network, we can build an efficient wireless network fast, improve the coordinate ability and efficiency among the base stations, and finally realize light network easy to deploy and maintain [6-8].
Cluster-based ad hoc network always apply to mobile communication scene with deficient base installation, which can constitute large-scale ad hoc networks by many clusters or many subnetworks. A large-scale ad hoc network is not dependent on base installation, can transmit flexible multihop, support real-time dynamic topology, and can be selforganization and self-recover. However, it is faced with the increasing observability of the network overhead, the longer the networking time, and the problem of internetwork interference as well as transmission interruption because of the network nodes increasing $[9,10]$. In cluster-based ad hoc networks, the scale of networks can be reduced to a certain extent, and then the problem of network synchronizing, resource scheduling, and the network routing can be resolved in local scope. After that, the multiple clusters or subnetworks with restricted scale can compose a large-scale ad hoc network.

In order to enhance the MAC protocol performance of the ad hoc network, a cooperative MAC protocol with relay node fast selection is proposed in [11]. The cooperative MAC protocol can choose the optimal relay nodes by priority 
difference and $k$-times competition and can decrease network overhead as well as improve transmit efficiency through introducing grouping mechanism. However, it is not considered the problem of efficiency reduce because of the network scale enlargement. A medium access scheme for vehicular ad hoc networks which is based on clustering of the vehicles is proposed for the effect of hidden stations [12]. In the Cluster-Based Medium Access Control Protocol, the bandwidth of the members in the cluster is distributed by the cluster head. CBMAC protocol is designed for one-hop communication in order to reducing intercluster interference, therefore, it is difficult to adapt to a wide range of application scenarios for ad hoc network. In [13], in order to overcome the lack of specialized hardware for infrastructure and the mobility to support network stability and channel utilization, Almalag et al. present a MAC algorithm for vehicular ad hoc networks using a new method for TDMA slot reservation based on clustering of vehicles; the algorithm is aimed at decreasing collisions and packet drops in the channel as well as providing fairness in sharing the wireless medium and minimizing the effect of hidden terminals. In order to improve the network throughout and reduce the end-toend transmission delay, a multichannel cluster distributed MAC protocol based on machine learning is proposed in [14]. Aiming at the problem of spectrum resource, the cluster head can monitor the intracluster transmission and then learn the transmit throughput of every member. Finally, the cluster head can allocate the optimized TDMA time-slots allocation result for every member in the cluster.

In this paper, MAC protocols in cluster-based ad hoc networks are researched for small-scale dense networking scene and large-scale extended coverage networking scene. Furthermore, the time-slot structure, the network synchronization algorithm, and the dynamic time-slot allocation algorithm are proposed for the cluster-based ad hoc networks. Finally, the performance of packet delivery rate and transmission delay is analyzed through simulation, and then the effectiveness of the MAC protocol of the cluster-based ad hoc networks is verified.

\section{Related Works}

Resource scheduling, clock synchronization, and adoptive transmission are mainly realized in the MAC layer protocol of MANET. Wireless resource allocation is one of the key problems in MANET. When the outside environment changes, the transmission quality between network nodes frequently changes, too. Efficient utilization of the wireless channel resource is the primary mission of MAC protocol, which can decrease data transmission conflict and secure network access fairly for network nodes $[15,16]$. According to the difference of channel access strategy, the MAC layer protocol can be divided into competition protocol and allocation protocol [17].

In the competition protocols, the nodes use channel resource through competitive mode. The implementation of competition protocols is always relatively simple, and it works well when the number of nodes is small and the network overhead is low, this is because of the adapt to topology changes at this present. However, when nodes or the network overhead increase, the probability of conflict for wireless channel resource is using increase significantly at the same time. Thus, the channel resource utilization will drop sharply, and the network throughput is decreased simultaneously $[18,19]$.

In the allocation protocols, the wireless resource always used time-division by time-slot as a unit. Therefore, we can achieve conflict-free data communication because the nodes are using their own allocated time-slots. The allocation protocols can improve the space reuse rate of the channel resource, so we can achieve a higher packet delivery ratio, higher information reliability, lower propagation delay time, and effetely data transmission for QoS. According to the difference of channel allocation algorithm used for the network, it can be divided into fixed time-slot allocation algorithm $[20,21]$ and dynamic time-slot allocation algorithm [22-24].

(a) In fixed time-slot allocation algorithms, the fixed time-slots are allocated to the fixed nodes, in other words, only one node can transmit with the fixed nods. Therefore, data transmission in the network is fair and collision-free, and the control overhead is small. However, it cannot reallocate the time-slots according to the traffic and cannot reuse the timeslots according to space relation. The result is that the valuable wireless resource is waste and cannot achieve higher throughput

(b) In dynamic time-slot allocation algorithms, the timeslots can be allocated as needed at a certain moment. When the nodes need to transmit data, the time resources will be allocated according to the current topology and a certain time-slot allocation algorithm. The overall performance of the network can be improved through the dynamic time-slot allocation algorithms, therefore, which are more and more attention in the current research. According to whether or not there is a central control node, the dynamic time-slot allocation algorithm can be further divided into two categories, one is the centralized dynamic time-slot allocation algorithms, and another is the distributed dynamic time-slot allocation algorithms. Just as its name implies, in the centralized dynamic time-slot allocation algorithms, there is a central control node which is responsible for the dynamic time-slot allocation, the central control node collects all of the business request among the whole network, and then carries out the action of the time-slot allocation, finally, the result is broadcast over the network. These algorithms possess the advantage of a good QoS guarantee, but the disadvantage of the short life cycle of the central control node. However, in the distributed dynamic timeslot allocation algorithms, every node calculates the result of time-slot allocation itself, in other words, all of the nodes are equal in status, but the good QoS is difficult to achieve

Ad hoc network generally has a large network scale and always needs to support voice, data, video, and other services; 


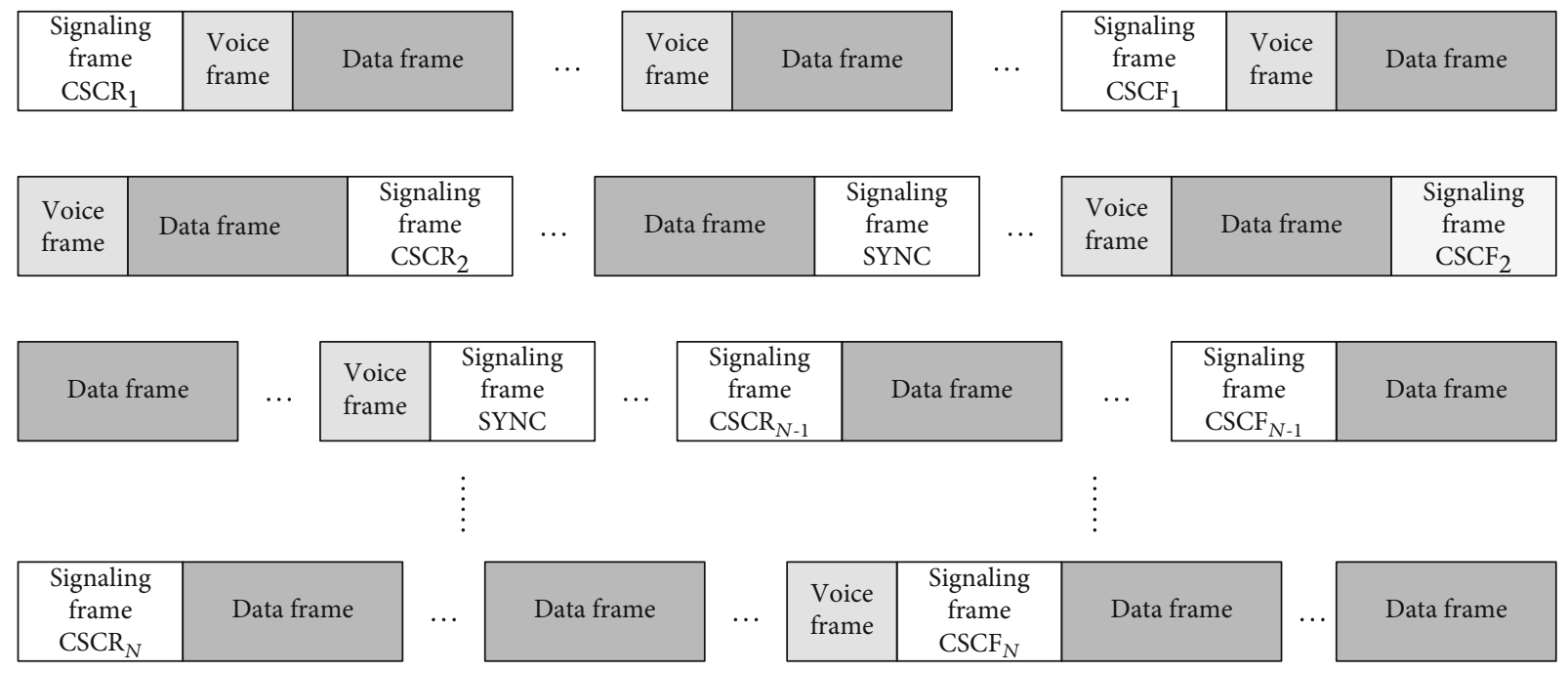

FIgURE 1: Time division in MAC protocol of our proposed scheme.

therefore, it needs a good QoS guarantee. Considering the flexibility of resource scheduling and the adaptive transmission, the centralized dynamic time-slot allocation algorithms are usually used in MAC protocol for ad hoc network. Based on this, we propose a MAC protocol in this paper, and the details will be provided in the following.

\section{MAC Protocol Design for Cluster-Based Ad Hoc Network}

MAC protocol for cluster-based ad hoc network mainly includes intracluster MAC protocol and intercluster gateway node selection. In which, intracluster MAC protocol mainly includes time-slot structure design, clock synchronization algorithm, and time-slot scheduling algorithm.

3.1. MAC Protocol Based on Scheduling of Cluster Head. The space, time, and frequency resources in the network can be managed in MAC protocol based on scheduling of cluster head, so that collision and retransmission between nodes will be avoided. Therefore, the protocol is especially suitable for the ultradense network and multiservice simultaneously transmission scenes. In this paper, a MAC protocol based on scheduling of cluster head is proposed, which can effectively reduce the network transmission delay through decentralized designing the different functional time-slot. Finally, the efficiency of the packet delivery rate is improved.

3.1.1. Time-Slot Structure Design. Three time slots including signaling time-slot, voice time-slot, and data time-slot are designed in the MAC protocol for cluster-based ad hoc network in this paper, which alternate between a complete scheduling cycle, as shown in Figure 1. Where the signaling time-slot is mainly used for network synchronization and dynamic resource scheduling, the voice time-slot is reserved for voice service to ensure real-time and efficient transmission, and the data time-slot is used for data service transmission.
As shown in Figure 1, a complete scheduling cycle includes several signaling time-slots, voice time-slots, and data time-slots. Where according to different transmission functions, signaling time-slots can be divided into SYNC frame, CSCR frame, and CSCF frame.

(1) SYNC frame which can be transmitted randomly is used for time synchronization. SYNC frame carries time information, node information, frequency information, bandwidth information, and so on. The workflow of the SYNC frame is shown in Algorithm 1

(2) CSCR frame always carries CSCR information, synchronization information, and topology information, the number of which is $N$. Normally, the CSCR frame has three functions: one is for nodes to send services requests to cluster head, the second is to send synchronization information to establish and maintain network time synchronization, and the third is to send network topology information and maintain network topology

(3) CSCF frames always carry CSCF information and synchronization information, the number of which is $N$. Normally, the CSCF frame has two functions: one is for cluster head to broadcast time-slot allocation results to intracluster nodes and the other one is to send synchronization information to establish and maintain network time synchronization

(4) Voice frame is mainly used to carry voice services, which generally uses the lowest bandwidth in the physical layer and the minimum transmission rate to ensure transmission reliability. The voice frames is usually dynamically allocated to the corresponding nodes according to the service application in every scheduling cycle

(5) Data frame is mainly used to carry data services. In order to adapt different transmission scenes, the 
The transmitting and receiving workflow of SYNC frame for time-slot $=1: n$

if (is-sending-timeslot $($ time-slot $)==1$ )

send the data packet;

else if some data packet arrives, receive this packet;

else if there are SYNC frames in the sending buffer, send this SYNC frames;

else if there are no SYNC frames in the sending buffer, end if plan to receive the SYNC frames;

end for

Algorithm 1: The transmitting and receiving workflow of SYNC frame.

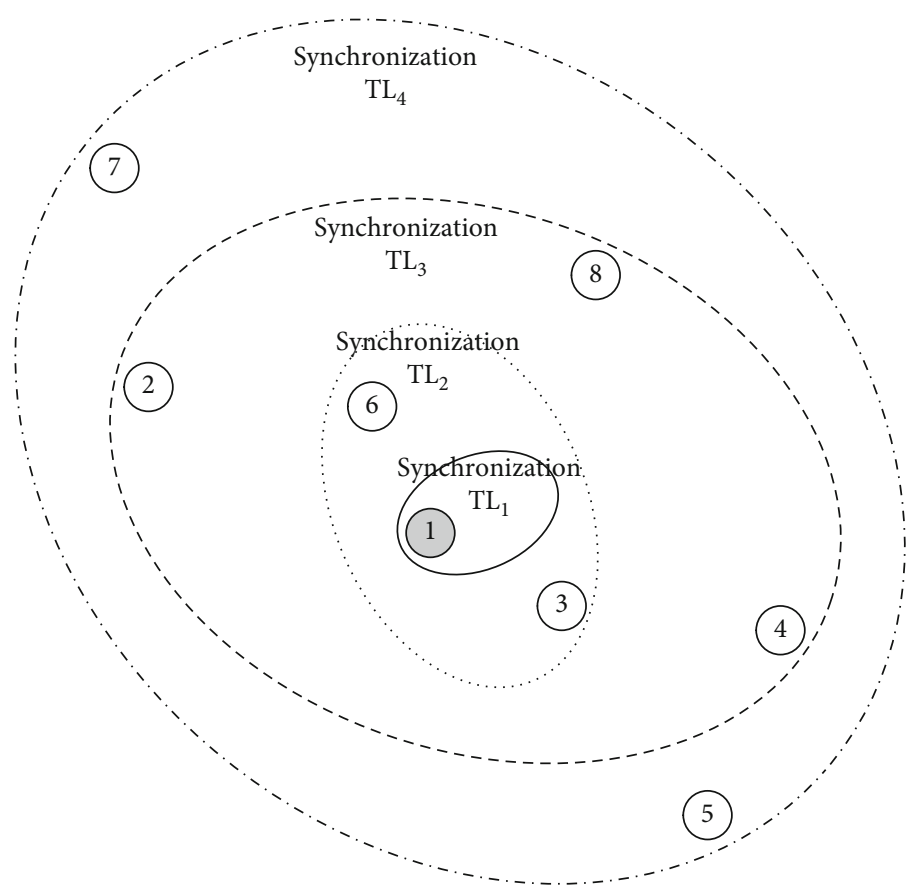

FIGURE 2: The schematic diagram for the spread of network synchronization.

physical layer can provide a variety of signal bandwidth, a variety of transmission rate for MAC protocol scheduling. The data frames are usually dynamically allocated to the corresponding nodes according to the service application in every scheduling cycle

\subsubsection{Network Synchronization Algorithm Based on Clock} Spread of the Sponsorship Node. Network synchronization is mainly realized by SYNC frames. Generally, any nodes can be set as the synchronization source node of the network. Node 1 in this paper is set as the synchronization source node. The scheme of network s synchronization is shown in Figure 2.

As shown in Figure 2, in the initial phase of network synchronization, node 1 broadcasts synchronous detection information including the transmission time T1, the node ID, and the node clock level TL1 in SYNC time-slot. Node 3 and node 6 which in the one-hop communication range of node 1 can determine its reference node and clock level through monitoring synchronous detection information of node 1. In Figure 2, the clock level of node 3 is updated to TL2, and node 1 is added to the local link information table. Then, node 3 can complete synchronization with the reference node, and so on. The network synchronization algorithm based on clock spread of the sponsorship node (as shown in Figure 3) is as follows:

(1) Synchronization sponsor nodes, such as node 1, broadcast synchronous detection information in SYNC time-slots, the transmission time is marked as $\mathrm{T} 1$

(2) Synchronization nodes, such as node 3, whose local record node $\mathrm{ID}=3$ receive the synchronous detection information of node 1 . And the synchronous detection information includes the source node $\mathrm{ID}=1$ and the transmission time T1. Then, node 3 sets its 


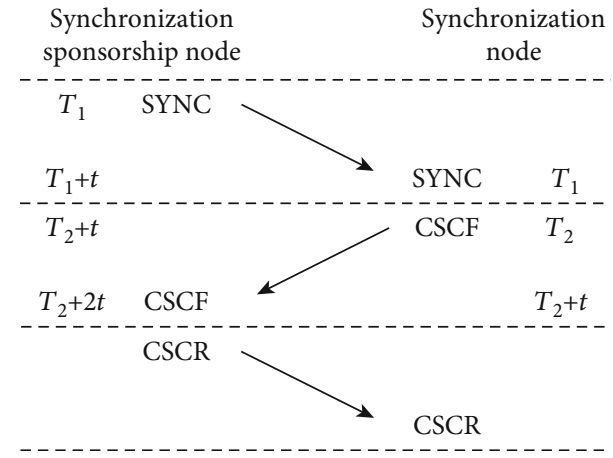

FIgURE 3: The schematic diagram of our proposed scheme for network synchronization a based on clock spread of the sponsorship node.

local time as T1. When the coarse synchronization is completing, the time difference between node 1 and node 3 is transmission delay $t$. After that, node 3 updates the local synchronous level to TL2

(3) Synchronization nodes, such as node 3, send synchronous information frame to synchronization sponsor node (node 1) in CSCF3 time-slots. The synchronous information frame is unicast, and the timestamp carried by CSCF3 is T2, the time when the sponsor node receives synchronous information frame is $T 2+2 t$. Therefore, the sponsor node can calculate the value of transmission delay $t$

(4) Synchronization sponsorship nodes, such as node 1, broadcast synchronous results in CSCR1 time-slots, and the CSCR 1 carries transmission delay $t$ of the two nodes. Synchronization node 3 extracts $t$ from the CSCR1 information, and then adjusts its own clock. Finally, node 3 accesses synchronization with the network

(5) The network synchronization is maintained periodically through a similar algorithm after the nodes completing synchronization with the network

3.1.3. Dynamic Time-Slot Allocation Algorithm Based on Schedule-Tree. The typical schedule-tree algorithms conclude the minimum hop schedule-tree algorithm, breadth first schedule-tree algorithm based on graph theory, and interference sensing schedule-tree algorithm based on concurrent transmission. All of these algorithms mainly consider the following four factors: hops between the parent node and the cluster head, distance between the trunk node and the cluster head through parent node, node ID of the parent node, and the multihop interference value of the parent node. The existing schedule-tree algorithms are usually executed when selecting sponsor node in the process of new nodes accessing to the network, and the selection of sponsor node is random. At this time, there is no definite criterion for the schedule-tree structured, and the calculation results of different nodes at different times have great deviation [25].
In view of the shortcomings of the existing schedule-tree algorithms, a proved schedule-tree algorithm based on cluster head and definite sponsor nodes is proposed in this paper. The construction of schedule-tree is completed by the cluster head through the interaction of signaling frames between cluster head and other nodes after the process of new nodes accessing to the network. In this way, the network conflicts and overhead are significantly reduced, and the network fairness has been improved. Furthermore, the selection basis of specific sponsor nodes is further clarified in this algorithm, so that more reasonable and optimized schedule-tree can be constructed.

The proved schedule-tree algorithm based on cluster head and definite sponsor nodes mainly considers the following five factors: hops between the potential parent node and the cluster head, total number of the multihop child nodes of the potential parent nodes, multihop interference value of the potential parent nodes, the SNR between the potential parent node, and the sponsor node. The detailed algorithm flow is as follows:

(1) The cluster head broadcast CSCF information, which carry the structure of existing schedule-tree and all the nodes information of the schedule-tree

(2) The child nodes of the cluster head save the scheduletree information after receiving the CSCF information of the current schedule-tree structure. The node will not continue to transmit CSCF information if it has no child node, but send CSCR information to apply for service time-slot in the corresponding time slot according to the allocation results. Conversely, the node will transmit CSCF information in the corresponding schedule time-slot according to their own index numbers if it has child nodes, at this time, the node will also send CSCR information according to service requirements in this scheduling cycle

(3) When completing the network topology updating, the cluster head adds the nodes in the network but not in the schedule-tree to the schedule-tree according to the network topology and the current schedule-tree

Considering the dynamic changes of services in the network, the dynamic time-slot resource allocation algorithm based on business requirements can effectively improve the efficiency of resource utilization. So the QoS requirements of different services can be contended. In the dynamic time-slot allocation algorithm based on schedule-tree, the nodes can transmit information after resources request and authorization are adopted. That is, only these nodes with service requirements can apply for time-slot resources. According to the existing time-slot resources allocation status, the cluster head responds to the resource application of nodes and then allocates the corresponding time-slots to realize the ondemand allocation of time-slot resources. The dynamic timeslot allocation algorithm based on schedule-tree mainly includes three processes: resource request, resource authorization, and data transmission, as shown in Figure 4.

In the resource request stage, the nodes in the network do not transmit immediately when receiving data from the 


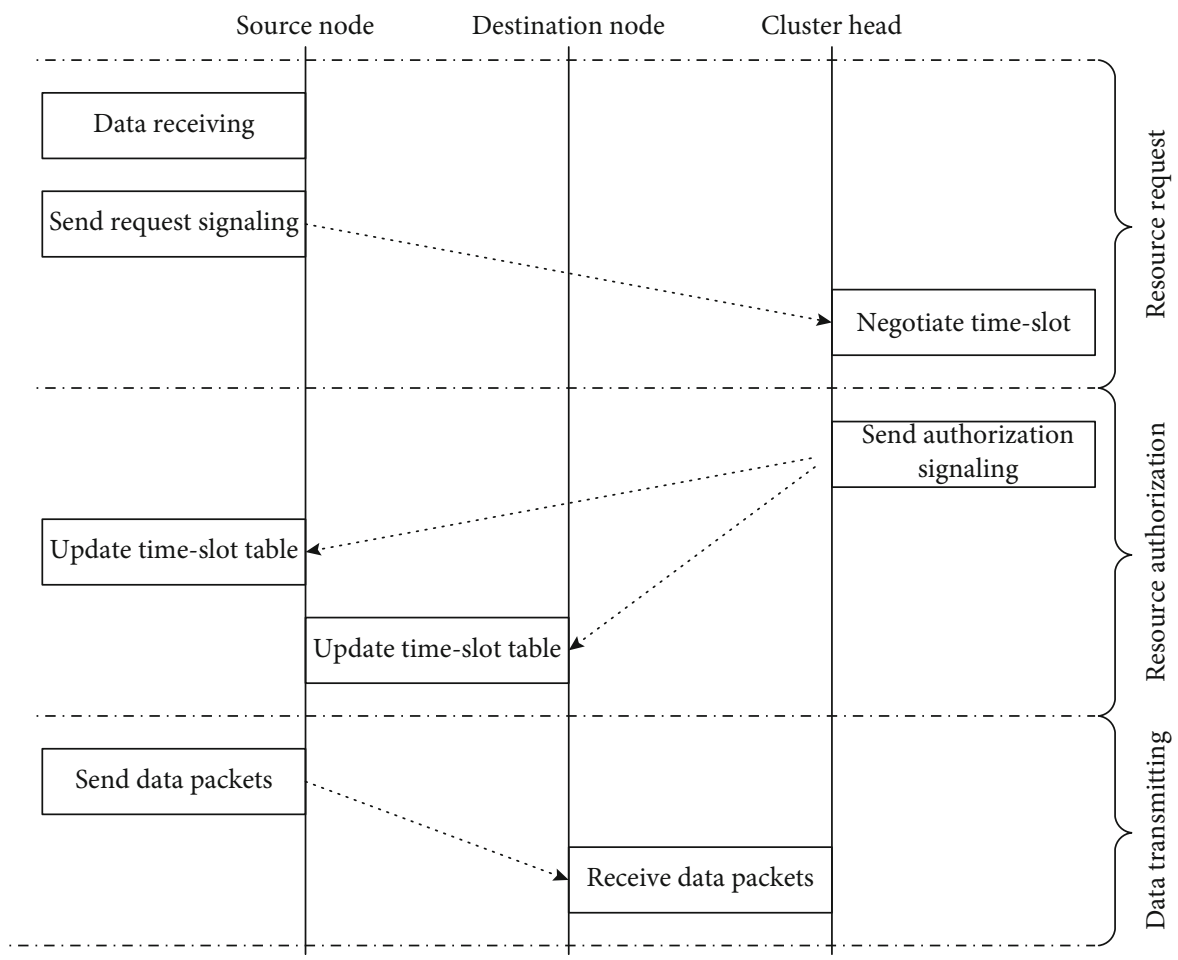

FIGURE 4: The time-slot resources scheduling flow chart of our proposed scheme.

application layer, but need to send the request message CSCR to the cluster head firstly, which carries the corresponding requirements information of the service QoS. When receiving the application information, the cluster head will allocate the time-slot resources for the corresponding service of nodes according to the information of network topology, network load, and service priority. In the resource authorization stage, cluster head will broadcast the time-slot resources allocation results to all nodes intracluster and authorize the time-slot resources. When receiving the authorization signaling information, the nodes in the network acquire their transmitting and receiving time-slots and then update the local time-slot table. In the data transmission stage, when system time arriving the transmitting or receiving time-slots, the corresponding nodes can transmit or receive data.

In the dynamic time-slot allocation algorithm based on the schedule-tree proposed in this paper, the time-slot allocation results will be updated every complete scheduling cycle. Both the transmitter and the receiver can transmit or receive data according to the time-slot allocation results. The proposed algorithm can effectively avoid network collision when the network topology and schedule-tree are relatively stable in a scheduling cycle.

3.2. Simulation Analysis. To verify the performance, we will give the simulation analysis of our algorithm. The tool we used is NS2 (Network Simulator version 2), which is an open-source tool for simulating and developing networks. Assume the number of nodes in a single cluster is $N=32$, the whole network has 160 nodes, a complete scheduling cycle is $1 \mathrm{~s}$, the length of signaling time-slot, voice time-slot, and data time-slot are $4 \mathrm{~ms}, 2 \mathrm{~ms}$, and $8 \mathrm{~ms}$, respectively.
The network nodes are randomly distributed in $5 \mathrm{~km} \times 5 \mathrm{~km}$ in small-scale dense networking scene and distributed in 250 $\mathrm{km} \times 250 \mathrm{~km}$ in large-scale extended coverage networking scene.

3.2.1. Simulation Analysis for Small-Scale Dense Networking Scene. Assuming that the nodes transmit along line of sight in a small-scale dense networking scene, the moving speed is randomly distributed between 0 and $30 \mathrm{~km} / \mathrm{h}$ and the direction is random, its typical network topology is shown in Figure 5.

Set the simulate time to $10 \mathrm{~min}$ in NS2, the package delivery rate and the probability distribution of transmission delay between any two nodes obtained by the simulation results are shown in Figures 6 and 7, respectively. As shown in Figure 6, the package delivery rate tends to be stable about $20 \mathrm{~s}$, because it is the network establishment stage in the first $20 \mathrm{~s}$, while the network has not completed synchronization at this stage. Finally, the packet delivery rate is stable at about 99\%. Analysis shows that the reason of packet loss is the instantaneous routing chosen bias due to the random movement of nodes, which can be avoided in application by application layer, MAC layer, or physical layer retransmission. It can be seen from the probability distribution map of transmission delay in Figure 7, most data packets can be delivered in a short time delay because the simulation environment is a small-scale dense networking scene and the nodes can reach each other in one hop. Therefore, the transmission delay is small in this scene.

3.2.2. Simulation Analysis for Large-Scale Extended Coverage Networking Scene. In a large-scale extended coverage 




FIGURE 5: The topology diagram for large-scale extended coverage networking.

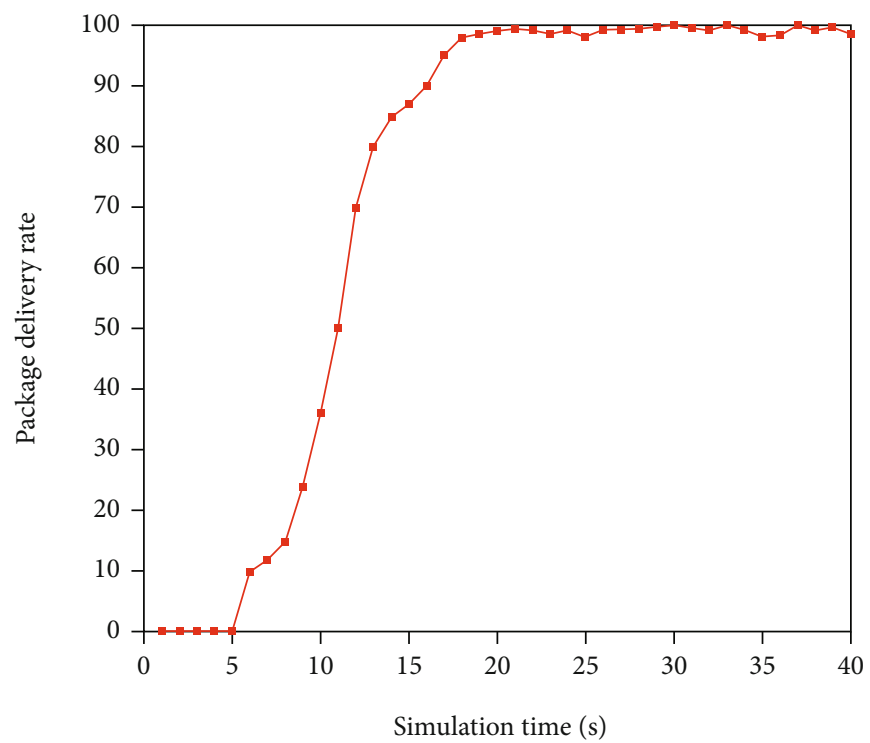

FIGURE 6: The performance evaluations for package delivery rate of our proposed scheme in small-scale dense networking scene.

networking scene, which can cover the entire area of $250 \mathrm{~km}$ $\times 250 \mathrm{~km}$ in the simulation, there are both airborne nodes and ground nodes. Assuming that airborne nodes are hovering, the moving speed of ground nodes is randomly distributed between 0 and $60 \mathrm{~km} / \mathrm{h}$, and the direction is random, its typical network topology is shown in Figure 8.

Set the simulate time to $10 \mathrm{~min}$ in NS2, the package delivery rate and the probability distribution of transmission delay between any two nodes obtained by the simulation results are shown in Figures 9 and 10, respectively. As shown in Figure 9, the package delivery rate tends to be stable about $35 \mathrm{~s}$. Therefore, the network establishment time is not more than $35 \mathrm{~s}$, and it does not increase much relatively to the small-scale dense networking scene, due to there are some airborne nodes, and the other node intracluster can reach one of the airborne nodes one-hop. So, the network synchronization time is fast. Because the airborne node is the only alternative gateway, network establishment time intercluster is also relatively fast.

It can be seen from the probability distribution map of transmission delay in Figure 10, most data packets can be delivered in a short time delay, because there are airborne 


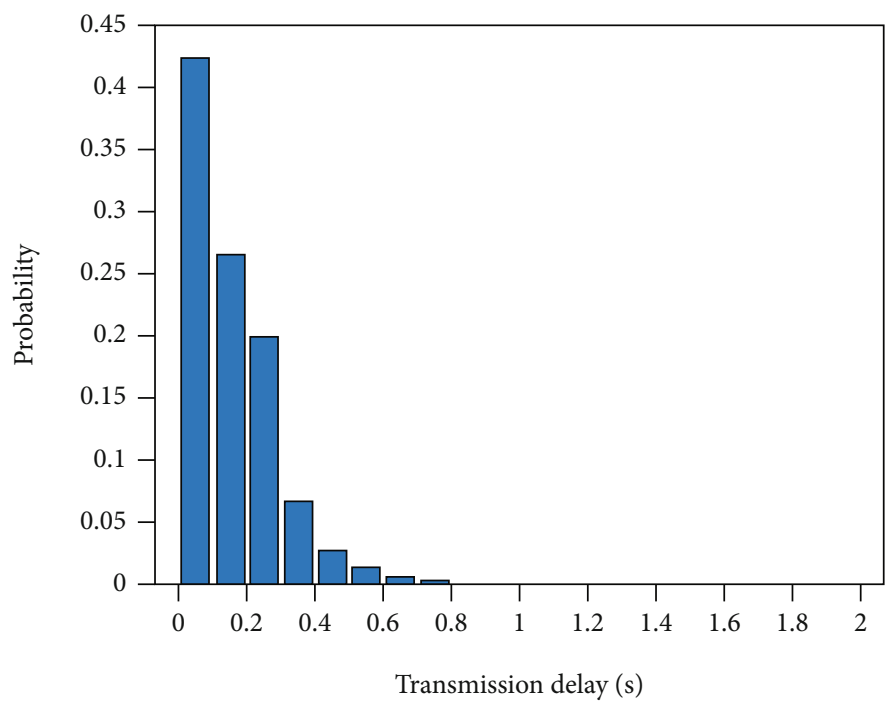

FIGURE 7: The performance evaluations for transmission delay of our proposed scheme in small-scale dense networking scene.

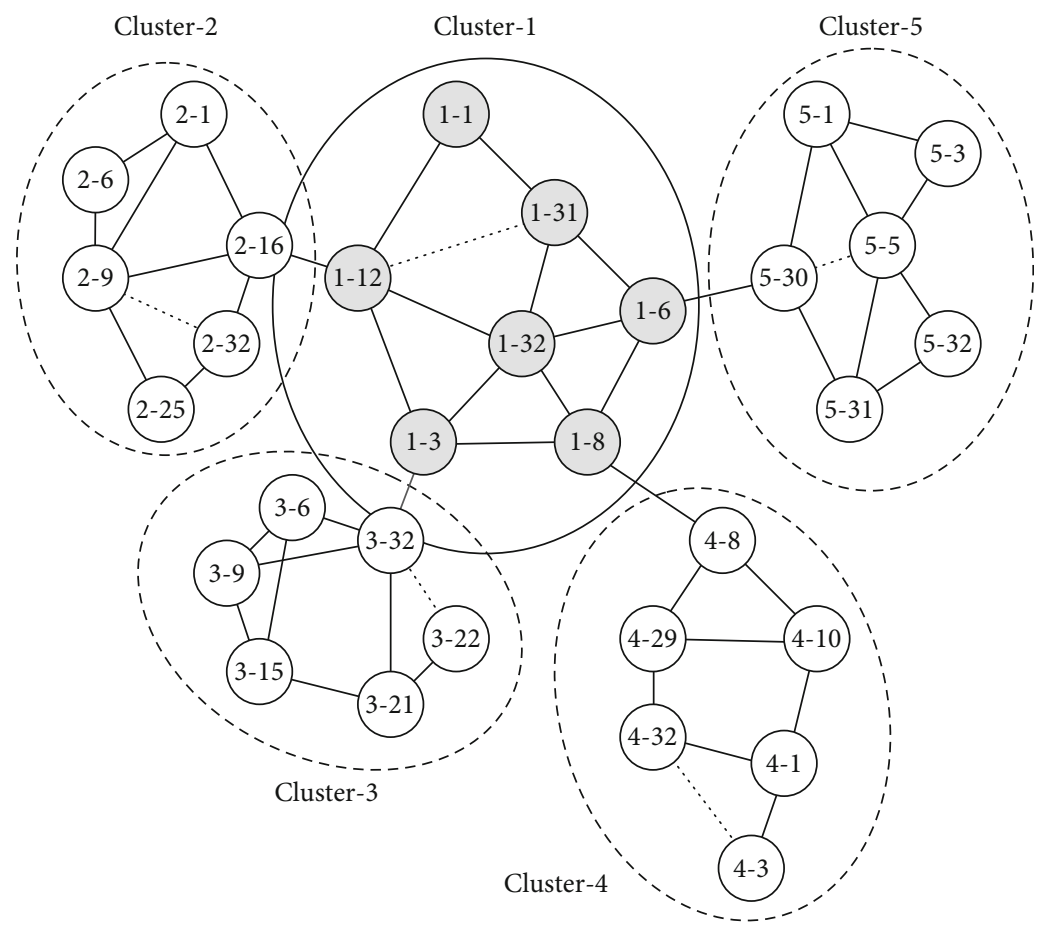

FIGURE 8: The topology diagram for small-scale dense networking.

nodes in this scene, and the communication relay times between any two nodes are no more than two-hops.

\section{Intercluster Communication Design for Cluster-Based Ad Hoc Network}

In cluster-based communication networks, resource manager or multihop communication for intercluster always depend on the gateway nodes, which is harmful to achieve load balancing and is harmful to balance the energy consumption between nodes. Therefore, the gateway nodes selection algo- rithms need to focus on the problem of how to reduce overhead of intercluster communication control information and how to improve the reliability of intercluster communication when the network topology changes, as well as how to achieve network load balancing, and so on [26, 27]. In summary, a dynamic gateway selection algorithm based on link stability is proposed in this paper to resolve the problems mentioned above.

4.1. Dynamic Gateway Selection Algorithm Based on Link Stability. The gateway nodes are selected according to the average received signal level and the communication link 


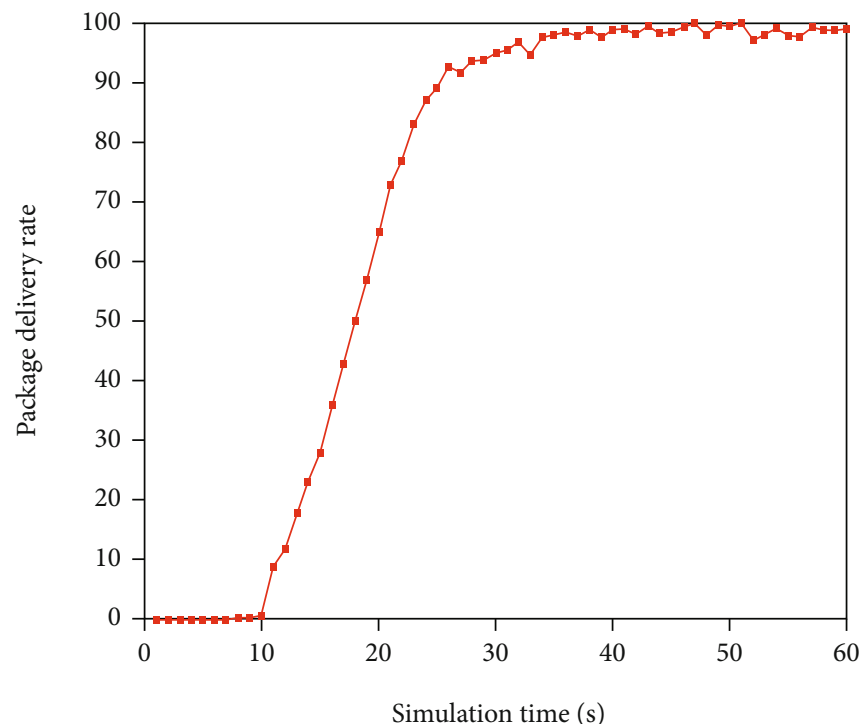

FIgURE 9: The performance evaluations for package delivery rate of our proposed scheme in a large-scale extended coverage scene.

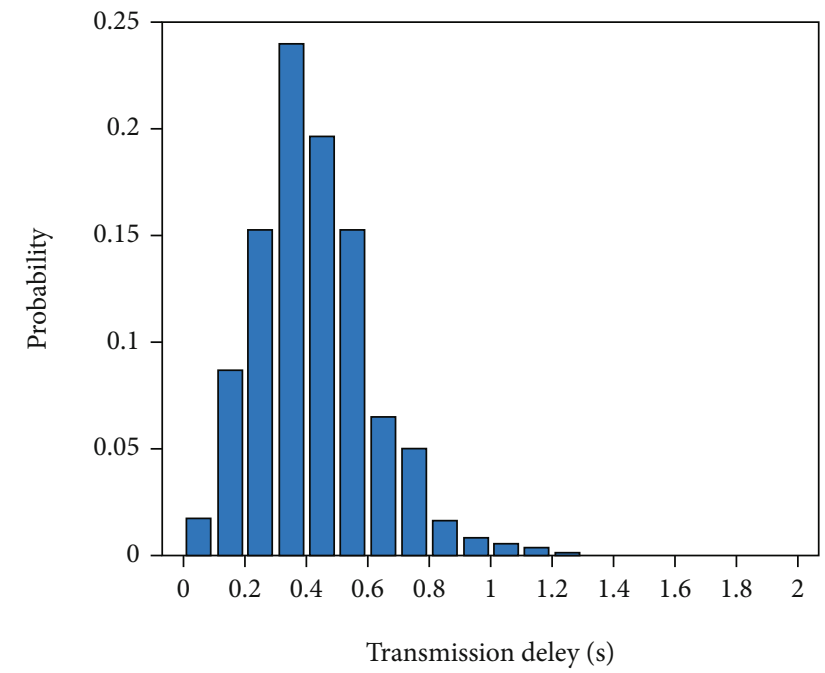

FIGURE 10: The performance evaluations for transmission delay of our proposed scheme in a large-scale extended coverage scene.

statistics in the dynamic gateway selection algorithm based on link stability. The communication link statistics can be expressed by the number of communication interruptions in a certain period, and the average received signal level can be predicted by the free space propagation model:

$$
P_{r}(d)=\frac{\left(P_{t} G_{t} G_{r}^{2}\right)}{\left[(4 \pi)^{2} d^{2} L\right]}
$$

where $P_{r}$ and $P_{t}$ are the receiving power and the transmitting power, respectively, $G_{r}$ and $G_{t}$ are the antenna gain of receiver and transmitter, respectively, $d$ is the distance between the receiving and transmitting nodes, and $L$ is system loss rate. The gateway nodes are selected mainly accord- ing to the communication link statistics when the network topology changes dramatically, and they are selected mainly according to the average received signal level under a complex electromagnetic environment.

Assume that the frequency between cluster 1 and cluster 2 is orthogonal, namely, cluster_1 and cluster_2. The cluster number will be transmitted in the SYNC signaling. The process of dynamic gateway node selection is described as follows.

(1) Any node in the cluster is labeled as a reserved gateway node and reports it to the cluster head through the CSCR frame when receiving SYNC information transmitted by other clusters

(2) The cluster head selects the intracluster transmitter gateway node according to the dynamic gateway selection algorithm based on link stability and then transmit to the corresponding nodes by CSCF frame

(3) The gateway nodes declare to other clusters the information by SYNC frame after it was confirmed as the intracluster gateway node

(4) The nodes in the adjacency cluster apply to the cluster head to become a receiver gateway node by CSCR frame when receiving the SYNC frame of other clusters

(5) The receiver gateway nodes are selected and confirmed by the cluster head according to the dynamic gateway selection algorithm based on link stability, and then CSCF frame with the receiver gateway nodes information will be transmitted to corresponding nodes

4.2. Intercluster Communication Analysis for Cluster-Based Ad Hoc Network. Next, take the dual-channel intercluster as an example, the realization of intercluster communication for cluster-based ad hoc network will be analyzed. 


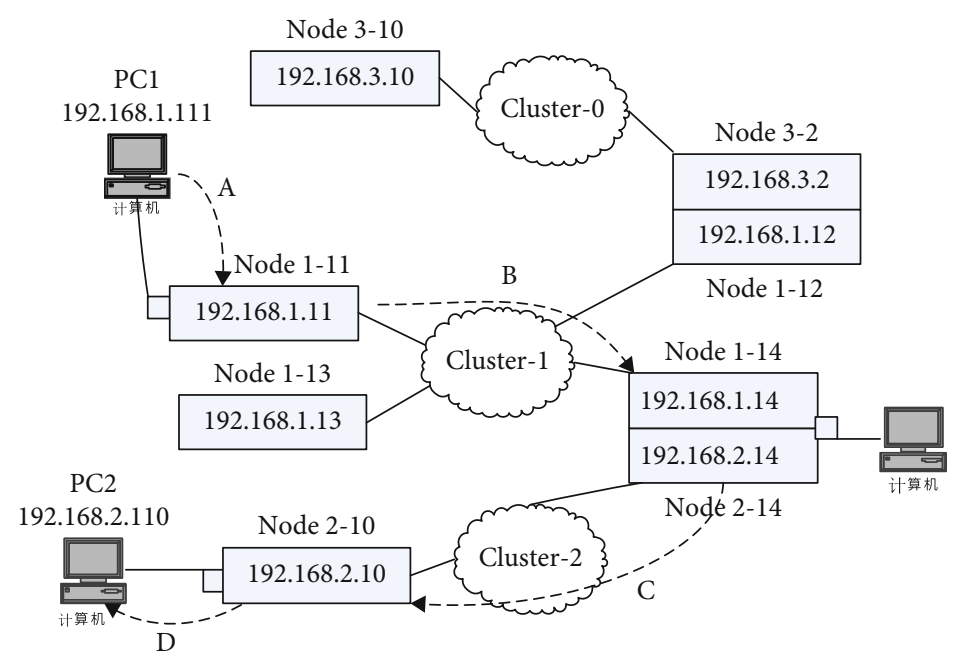

FIGURE 11: Intercluster communication diagram through dual-channel gateway nodes.

Considering a cluster-based ad hoc network as shown in Figure 11, in which three clusters are connected by gateway nodes. The service terminals (laptop, etc.) connected to the nodes need to set the node IP address as the default gateway, and the IP address of the service terminal needs to keep the same network segment as the default gateway. As shown in Figure 11, node 1-12, node 1-14, node 2-14, and node 3-2 are gateway nodes selected by the dynamic gateway selection algorithm based on link stability selects. When data packet transmitting from $\mathrm{PC} 1$ to $\mathrm{PC} 2$, the default gateway of $\mathrm{PC} 1$ is set as 192.168.1.111, and the default gateway of PC2 is set as 192.168.2.110. The detail process is described as follows.

(1) The node connected with PC1 will transmit data to the default gateway, which is node $1-14$, when recognizing that the IP address of PC2 is not in the same cluster. The destination MAC address and IP address of data packets is the MAC address and IP address of node 1-11 in link A

(2) After receiving data packets in the network layer of node 1-11, it firstly confirm that the destination IP is one node intercluster, and secondly find the destination node intracluster whose IP address is 192.168.1.14 through searching in the forwarding table. And then it can replace the destination MAC address of node 1-14 through searching in the IPMAC, so the data can be transmitted to node 1-14

(3) When receiving the data packets, node 1-14 firstly confirm that which are intercluster communication data and then transmit the data to 192.168.2.14 according to network number 2

(4) Node 2-14 modifies the destination MAC address as the MAC address of PC2 when confirming that the data packet is intercluster. Then, the data can be transmitted to node $2-10$ by the intracluster routing

(5) The data is sent to Ethernet interface by MAC layer of node $2-10$

\section{Conclusions}

One of the effective ways to realize large-scale networking is a cluster-based ad hoc network, which can effectively reduce the network overhead and improve the network stability by clustering and cascading. The MAC protocol for clusterbased ad hoc network needs to realize intracluster network synchronization and resource schedule, at the same time, which also needs to achieve intercluster dynamic gateway selection and whole network synchronization.

The MAC protocol based on scheduling of cluster head proposed in this paper especially suitable for ultradense network and multiservice simultaneous transmission scenes, which can avoid collision and retransmission between nodes to the maximum through unified management of the time, space, and frequency resources for intracluster and intercluster. The time-slots of signaling, voice, and data designed in the MAC protocol based on scheduling of cluster head are alternated in a complete scheduling cycle, so the network transmission delay can be effectively decreased. The clock of the sponsorship nodes can be rapidly spread out by the SYNC, CSCF, and CSCR frames of the signaling time-slots, which can reduce the network synchronization time and improve the network construction speed. Furthermore, the selection basis of specific sponsor nodes is further clarified, and then the schedule-tree can be calculated by the cluster head. Therefore, the schedule-tree can be spread out in the whole network by the CSCF and CSCR frames of the signaling time-slots, so, the network conflict can be reduced, and the network fairness can be improved.

One of the key factors for intercluster communication is the gateway nodes. The selection of gateway nodes may affect the intercluster communication delay, transmission efficiency, stability, and reliability. The communication link quality and network stability are both considered in the dynamic gateway selection algorithm based on link stability proposed in this paper, so that the algorithm can be applied to different scenes. Finally, taking three ad hoc network clusters as example, detailed analysis and production of the interclusters communication flow for 
cluster-based network are carried out. In summary, both intracluster and intercluster communication are fully considered in the MAC protocol for cluster-based ad hoc network. Therefore, the protocol proposed in this paper has broad application prospects.

\section{Data Availability}

The data used to support the findings of this study are available from the corresponding author upon request.

\section{Conflicts of Interest}

The authors declare that they have no conflicts of interest.

\section{Acknowledgments}

This work was supported by the National Natural Science Foundation of China under Grant no. 61971162.

\section{References}

[1] Q. Ye and W. Zhuang, "Token-based adaptive MAC for a twohop internet-of-things enabled MANET," IEEE Internet of Things Journal, vol. 4, no. 5, pp. 1739-1753, 2017.

[2] D. J. Persis and T. P. Robert, "Review of ad-hoc on-demand distance vector protocol and its swarm intelligent variants for Mobile Ad-hoc NETwork," IET Networks, vol. 6, no. 5, pp. 87-93, 2017.

[3] A. M. E. Ejmaa, S. Subramaniam, Z. A. Zukarnain, and Z. M. Hanapi, "Neighbor-based dynamic connectivity factor routing protocol for mobile ad hoc network," IEEE Access, vol. 4, pp. 8053-8064, 2016.

[4] M. Bheemalingaiah, M. M. Naidu, D. S. Rao, and P. Vishvapathi, "Performance analysis of power-aware nodedisjoint multipath source routing in mobile ad hoc networks," in IEEE 7th International Advance Computing Conference, pp. 361-371, Hyderabad, India, 2017.

[5] A. Taha, R. Alsaqour, M. Uddin, M. Abdelhaq, and T. SABA, "Energy efficient multipath routing protocol for mobile adhoc network using the fitness function," IEEE Access, vol. 5, pp. 10369-10381, 2017.

[6] X. Liu and X. Zhang, "NOMA-based resource allocation for cluster-based cognitive industrial internet of things," IEEE Transactions on Industrial Informatics, vol. 16, no. 8, pp. 5379-5388, 2020.

[7] X. Liu, X. Zhai, W. Lu, and C. Wu, "QoS-guarantee resource allocation for multibeam satellite industrial internet of things with NOMA," IEEE Transactions on Industrial Informatics, vol. 17, no. 3, pp. 2052-2061, 2021.

[8] X. Liu and X. Zhang, "Rate and energy efficiency improvements for 5G-based IoT with simultaneous transfer," IEEE Internet of Things Journal, vol. 6, no. 4, pp. 5971-5980, 2019.

[9] B. N. Cheng, G. Kuperman, P. Deutsch, L. Mercer, and A. Narula-Tam, "Group-centric networking: addressing information sharing requirements at the tactical edge," IEEE Communications Magazine, vol. 10, pp. 145-151, 2016.

[10] Y. Cong, X. Zhou, and R. A. Kennedy, "Interference prediction in mobile ad hoc networks with a general mobility model,"
IEEE Transactions on Wireless Communications, vol. 14, no. 8, pp. 4277-4290, 2015.

[11] K. Liu, X. Y. Chang, and F. Liu, “A cooperative MAC protocol with rapid relay selection for wireless ad hoc networks," Computer Networks: The International Journal of Computer and Telecommunications Networking, vol. 91, pp. 262-282, 2015.

[12] Y. Gunter, B. Wiegel, and H. P. Grossmann, "Cluster-based medium access scheme for VANETs," in IEEE Intelligent Transportation Systems Conference, pp. 343-348, Seattle, Washington, USA, 2007.

[13] M. S. Almalag, S. Olariu, and M. C. Weigle, "TDMA clusterbased MAC for VANETs (TC-MAC)," in IEEE International Symposium on World of Wireless Mobile and Multimedia Networks, pp. 1-6, Norfolk, VA, USA, 2012.

[14] Q. Luo, C. Li, Q. Ye, T. H. Luan, L. Zhu, and X. Han, "CFT: a cluster-based file transfer scheme for highway VANETs," in 2017 IEEE International Conference on Communications, pp. 1-6, Washington, DC, USA, 2017.

[15] S. J. Wen and C. H. Huang, "Delay-aware cross-layer optimization method for FANET," Journal on Communications, vol. 39, no. 4, pp. 1-12, 2018.

[16] D. F. Zhao, B. H. Li, and S. M. Zheng, "Study of a polling systems with limited service," Journal of Electronics, vol. 19, no. 1, pp. 44-49, 1997.

[17] Q. Wang, S. Leng, H. Fu, and Y. Zhang, "An IEEE 802.11pbased multichannel MAC scheme with channel coordination for vehicular ad hoc networks," IEEE Transactions on Intelligent Transportation Systems, vol. 12, no. 3, pp. 449458, 2012.

[18] H. Saggar, Y. Jiang, B. Daneshrad, and P. Greg, “A concurrent CSMA MAC protocol for mobile ad hoc networks using beamnulling," in 2015 IEEE military communications conference, pp. 97-102, Tampa, FL, USA, 2015.

[19] W. Hu and X. Li, "Load adaptive MAC: a hybrid MAC protocol for MIMO SDR MANETs," IEEE Transactions on Wireless Communications, vol. 10, no. 11, pp. 3924-3933, 2011.

[20] L. Xing, Z.-X. He, and Z. Jing-Lun, "A minimum-jitter fixed timeslot allocation algorithm for TDMA tactical data link," System Engineering, vol. 30, no. 6, pp. 90-94, 2012.

[21] S. Rahmani, A. Khademzadeh, and A. M. E. Moghadam, "Time slot allocation algorithm based on TDMA protocol in wireless sensor networks," Journal of Advances in Computer Research, vol. 4, no. 4, pp. 61-71, 2013.

[22] A. B. M. A. Islam, M. J. Islam, N. Nurain, and V. Raghunathan, "Channel assignment techniques for multi-radio wireless mesh networks: a survey," IEEE Communications Surveys \& Tutorials, vol. 18, no. 2, pp. 988-1017, 2016.

[23] C. Song, G. Tan, and C. Yu, “APDM: an adaptive multipriority distributed multichannel MAC protocol for vehicular ad hoc networks in unsaturated conditions," Computer Communications, vol. 104, pp. 119-133, 2017.

[24] A. Raniwala, K. Gopalan, and T. Chiueh, "Centralized channel assignment and routing algorithms for multi-channel wireless mesh networks," ACM SIGMOBILE Mobile Computing and Communications Review, vol. 8, no. 2, pp. 5065, 2004.

[25] H. Artail and K. Mershad, "MDPF: minimum distance packet forwarding for search applications in mobile ad hoc networks," IEEE Transactions on Mobile Computing, vol. 8, no. 10, pp. 1412-1426, 2009. 
[26] S. H. Y. Wong, C. K. Chau, and K. W. Lee, "Managing interoperation in multi-organization MANETs by dynamic gateway assignment," in IEEE International Symposium on Integrated Network Management, pp. 129-136, Amsterdam, The Netherlands, 2011.

[27] S. Shruthi, "Proactive routing protocols for a MANET: a review," in 2017 IEEE International Conference on I-SMAC (IoT in Social, Mobile, Analytics and Cloud), pp. 821-827, Palladam, India, 2017. 